\title{
IST KANTS EWIGER FRIEDEN FÜR EWIG UNERREICHBAR?
}

\author{
Henny Blomme
}

\section{DIE INS UNENDLICHE FORTSCHREITENDE ANNÄHERUNG}

Kant beendet seinen philosophischen Entwurf Zum ewigen Frieden mit dem folgenden kurzen Absatz:

Wenn es Pflicht, wenn zugleich gegründete Hoffnung da ist, den Zustand eines öffentlichen Rechts, obgleich nur in einer ins Unendliche fortschreitende Annäherung wirklich zu machen, so ist der ewige Friede, der auf die bisher fälschlich so genannten Friedensschlüsse (eigentlich Waffenstillstände) folgt, keine leere Idee, sondern eine Aufgabe, die nach und nach aufgelöst, ihrem Ziele (weil die Zeiten, in denen gleiche Fortschritte geschehen, hoffentlich immer kürzer werden) beständig näher kommt. ${ }^{1}$

Nach dieser Passage ist, wenn eine gewisse Bedingung erfüllt ist, der ewige Friede kein bloßes Gedankending, sondern eine reelle Aufgabe für uns. Und durch das Lösen dieser Aufgabe kommt ihr Ziel näher. Bemerkenswert ist, dass der ,ewige Frieden' zugleich Aufgabe und Ziel der Aufgabe ist. Während wir uns aktiv dafür engagieren, den ewigen Frieden hier auf Erden zu realisieren, lösen wir nach und nach die Aufgabe, die er darstellt, und zugleich kommt das Ziel (verwirklichter ewiger Frieden) ,,beständig näher“".

Nun ist, so Kant, die Bedingung, die erfüllt sein soll, damit der ewige Frieden keine leere Idee sei und realistisch als Aufgabe dargestellt werden kann, die Verwirklichung des Zustandes ,eines öffentlichen Rechts“. Aber auch dieser ist zugleich Aufgabe und Ziel, und das Verwirrende ist, dass Kant nicht einmal die Möglichkeit eines erfolgreichen Abschlusses dieser Aufgabe - das endgültige Erreichen ihres Ziels - garantiert. Einerseits ist es Pflicht, den Zustand eines öffentlichen Rechts ,wirklich zu machen“, und es gibt auch „begründete Hoffnung“, dass man sich dieser Aufgabe mit einem gewissen Erfolg widmen kann. Andererseits aber bleibt es unklar, ob dieser Zustand endgültig oder „,nur in einer ins Unendliche fortschreitende Annäherung“ wirklich gemacht werden kann. Jedenfalls ist auch die Idee eines öffentlichen Rechts keine leere Idee. Das geht noch besser hervor aus dem, was Kant am Ende des dritten Definitivartikels schreibt:

[Die Idee] eines Weltbürgerrechts [ist] keine phantastische und überspannte Vorstellungsart des Rechts, sondern eine notwendige Ergänzung des ungeschriebenen Kodex sowohl des Staats- als Völkerrechts zum öffentlichen Menschenrechte überhaupt, und so zum ewigen Frieden, zu dem man sich in der kontinuierlichen Annäherung zu befinden nur unter dieser Bedingung schmeicheln darf. ${ }^{2}$

Wenn man von diesen Textpassagen ausgeht, stellt man fest, dass Kant die als Titel dieses Textes gewählte Frage nicht beantwortet: Wir können weder mit Sicherheit bestätigen, dass der ewige Frieden einmal wirklich bestehen wird, noch behaupten, dass er prinzipiell niemals wirklich werden kann. Das dürfte vielleicht auch die richtige Antwort sein. Nun gibt es aber in der Friedensschrift auch solche Passagen, in denen Kant davon auszugehen scheint, dass der ewige Frieden sowieso früher oder später Wirklichkeit wird. So lautet bekanntlich der Titel des ersten Zusatzes ${ }^{3}$ „Von der Garantie des ewigen Friedens“4. Um welche Art der Garantie geht es hier? Das werden wir zu erläutern haben (nämlich im dritten Abschnitt dieses Textes). Andererseits gibt es auch Passagen, durch die man den Eindruck bekommt, dass Kant lediglich über einen sogenannten Vernunftfrieden $^{5}$ spricht, im Sinne eines utopischen Ideals, also: ein Vernunftbegriff ohne objektive

1 ZeF B 111f., 08: 386.

2 ZeF B 46, 08: 360.

3 In der ersten Auflage einfach ,Zusatz“, da es dort noch keinen zweiten Zusatz gibt.

4 ZeF B 47, 08: 360 .

5 Diesen Terminus (den Kant meines Wissens nicht benutzt hat) entnehme ich dem Titel eines 1996 erschienenen Sammelbandes zum ewigen Frieden: Kodalle 1996. 
Realität. Von einem solchen Frieden anzunehmen, dass er realisiert werden kann, wäre dann einfach naiv. Im folgenden Abschnitt werde ich zunächst diese Ansicht, der ewige Frieden sei empirisch prinzipiell unrealisierbar, besprechen.

\section{DIE UNAUSFÜHRBARE IDEE}

In seiner Rezension der Friedensschrift hatte Fichte 1796 davor gewarnt, Kants Idee des ewigen Friedens lediglich als eine herzerwärmende Illusion aufzufassen:

[Die Beziehung] auf das Interesse des Tages, die Leichtigkeit und Annehmlichkeit des Vortrags und die anspruchslose Weise, mit welcher die in ihr vorgetragenen erhabenen, allumfassenden Ideen hingelegt werden, dürfte mehrere verleiten, derselben nicht die Wichtigkeit beizumessen, die sie unseres Erachtens hat, und die Hauptidee derselben für nicht mehr anzusehen als für einen frommen Wunsch, einen unmaßgeblichen Vorschlag, einen schönen Traum, der allenfalls dazu dienen möge, menschenfreundliche Gemüter einige Augenblicke angenehm zu unterhalten. ${ }^{6}$

In der Rechtslehre von 1797 scheint Kant jedoch selbst explizit zu behaupten, dass der ewige Frieden „eine unausführbare Idee“ sei. Im $\S 61$ schreibt er in der Tat Folgendes:

Da der Naturzustand der Völker, eben so wohl als einzelner Menschen, ein Zustand ist, aus dem man herausgehen soll, um in einen gesetzlichen zu treten: so ist, vor diesem Ereignis, alles Recht der Völker und alles durch den Krieg erwerbliche oder erhaltbare äußere Mein und Dein der Staaten bloß provisorisch, und kann nur in einem allgemeinen Staatenverein (analogisch mit dem, wodurch ein Volk Staat wird) peremptorisch geltend und ein wahrer Friedenszustand werden. Weil aber, bei gar zu großer Ausdehnung eines solchen Völkerstaats über weite Landstriche, die Regierung desselben, mithin auch die Beschützung eines jeden Gliedes endlich unmöglich werden muß, eine Menge solcher Korporationen aber wiederum einen Kriegszustand herbeiführt: so ist der ewige Friede (das letzte Ziel des ganzen Völkerrechts) freilich eine unausführbare Idee. Die politischen Grundsätze aber, die darauf abzwecken, nämlich solche Verbindungen der Staaten einzugehen, als zur kontinuierlichen Annäherung zu demselben dienen, sind es nicht, sondern, so wie diese eine auf der Pflicht, mithin auch auf dem Recht der Menschen und Staaten gegründete Aufgabe ist, allerdings ausführbar. ${ }^{7}$

In einer jüngst erschienenen Monographie zu Kants praktischer Philosophie wird diese Passage der Rechtslehre folgendermaßen kommentiert:

So unerreichbar der in der Idee eines ewigen Friedens gedachte Rechtszustand auch sein mag, es ist gleichwohl eine „auf dem Recht der Menschen und Staaten gegründete Aufgabe“, auf ihn fortwährend hinzuarbeiten. Denn wenngleich der ,ewige Friede“ nach Kant eine „unausführbare Idee“ ist, so sind die „politischen Grundsätze“, auf ihn in einer „,kontinuierlichen Annäherung“ hinzuwirken, ,allerdings ausführbar“. Das „Ideal einer rechtlichen Verbindung der Menschen unter öffentlichen Gesetzen“ ist eine notwendige und normative Leitidee, welche die politischen Akteure zu einer ,allgemeinen und fortdauernden Friedensstiftung“ verpflichtet. Dass die praktische Realisierung dieses Ideals nicht zu vollenden ist, ist für Kant daher letztlich von sekundärer Bedeutung. [...] Die Annäherung an ein empirisch unvollendbares Friedensideal ist [...] eine unbedingte moralische Vernunftforderung an die Politik. ${ }^{8}$

Nach dieser Interpretation wäre der ewige Frieden eine praktisch nicht zu realisierende Idee, obwohl wir zugleich die Pflicht hätten, uns derselben anzunähern. Daraus geht schon das Problematische dieser Deutung hervor: Ist es überhaupt möglich, etwas anzustreben, wovon man mit Sicherheit weiß, dass es niemals realisiert werden kann? Stärker noch: Wie können wir die Verpflichtung, ein bestimmtes Ziel anzustreben, ernsthaft als Verpflichtung hinnehmen, wenn es unmöglich ist, dass dieses Ziel jemals erreicht wird?

Ganz am Anfang des Anhangs zu seiner Friedensschrift schreibt Kant:

Die Moral ist schon an sich selbst eine Praxis in objektiver Bedeutung, als Inbegriff von unbedingt gebietenden Gesetzen, nach denen wir handeln sollen, und es ist offenbare Ungereimtheit, nachdem man diesem Pflichtbegriff

6 Fichte 1796, S. 81. Siehe Buhr / Dietzsch 1984, S. 93.

7 MS 06: 350 (Hervorhebung von H. B.).

8 Laschet 2011, S. 303. 
seine Autorität zugestanden hat, noch sagen zu wollen, dass man es doch nicht könne. Denn alsdann fällt dieser Begriff aus der Moral von selbst weg (ultra posse nemo obligatur). ${ }^{9}$

Einerseits ist es richtig, dass man nicht sicher sein muss, dass eine Handlung erfolgreich sein wird, um diese Handlung zu tun oder zu planen. Das gilt auch für solche Handlungen, die Pflicht sind: Man braucht keine theoretische Gewissheit darüber, dass das Ziel der Handlung erreicht werden kann, um das Handeln selbst als Pflicht anerkennen zu können. Andererseits darf das Handlungsziel nicht als bloße Illusion wahrgenommen werden. Ohne begründete, oder, wie Kant sagt, ,gegründete Hoffnung “"10, dass das Ziel (ewiger Frieden) realisiert werden kann, kann es auch keine Pflicht geben, auf es hinzuarbeiten.

Damit der ewige Frieden dennoch als Pflicht angesehen werden kann, muss also die Aussage, die man in der Rechtslehre findet - der ewige Frieden sei „eine unausführbare Idee“ -, weniger radikal verstanden werden. Dass das auch textuell richtig ist, geht aus dem Kontext dieser Aussage hervor. Bei näherem Hinschauen geht es Kant in der Tat gar nicht darum zu behaupten, dass die empirische Unmöglichkeit des Friedensideals sowieso schon feststeht. Vielmehr wird die Unmöglichkeit, den ewigen Frieden zu realisieren, in Verbindung gebracht mit einer bestimmten politischen Konstellation. Wie am Anfang dieses Textes schon klar wurde, ist die Realisierung des Friedens bedingt durch die Realisierung des Zustandes eines öffentlichen Rechts. Nun kann man sich, so Kant, die Realisierung des öffentlichen Rechts als Völkerrecht auf zwei Weisen vorstellig machen.

Zuerst bildet man sich die Vorstellung, dass ein weltumspannender Staat, eine Weltrepublik, gegründet werden muss. Kant schreibt, dass, wenn es darum geht, den ewigen Frieden zu realisieren, die Idee eines Weltstaats in thesi zwar richtig ist, in hypothesi aber verworfen werden müsse. ${ }^{11}$ Das wird meistens so verstanden, dass Kant die Idee eines Weltstaates theoretisch zwar für richtig, praktisch (d.h. hier: in der empirischen Realisierung) aber für problematisch hält. In der Tat beschreibt Kant wenigstens zwei Probleme, die mit der empirischen Realisierung der Idee eines Weltstaates einhergehen würden. Zum einen ist es nicht realistisch anzunehmen, dass verschiedene Völker sich jemals tatsächlich zu einem Volk zusammenschließen möchten. Zum anderen gäbe es bei der Realisierung des Weltstaates ein organisatorisches Problem, weil, „bei gar zu großer Ausdehnung eines solchen Völkerstaats über weite Landstriche, die Regierung desselben, mithin auch die Beschützung eines jeden Gliedes endlich unmöglich werden muß ${ }^{\text {“12 }}$. Die Alternative, mehrere kleinere Völkerstaaten zu gründen, muss ebenfalls fehlschlagen, weil „eine Menge solcher Korporationen [...] wiederum einen Kriegszustand herbeiführt" ${ }^{* 13}$. Gerade weil die Idee des Weltstaates in der Praxis Schwierigkeiten bereitet, müsse man sich mit der Realisierung eines ,Surrogats zufriedenstellen: eines Völkerbundes als „Friedensbundes“. ${ }^{14}$

Dass Kant in Zum ewigen Frieden eine theoretisch für richtig gehaltene Idee aus empirischpraktischen Gründen verwirft, erscheint jedoch problematisch, wenn man beachtet, dass er kurz davor noch eine solche Gegenüberstellung von empirisch-praktischen und theoretischen Gründen verworfen hatte. In der Tat wird im Gemeinspruch-Aufsatz (1793) die Möglichkeit einer relevanten Dissonanz zwischen Theorie und Praxis - gerade auch in Bezug auf das Völkerrecht - abgelehnt. Lesen wir nochmal, was Kant dort über die Idee eines Völkerstaates geschrieben hatte:

Die menschliche Natur erscheint nirgend weniger liebenswürdig, als im Verhältnisse ganzer Völker gegen einander. Kein Staat ist gegen den andern wegen seiner Selbstständigkeit, oder seines Eigenthums, einen Augenblick gesichert. [...] Nun ist hierwider kein anderes Mittel, als ein auf öffentliche mit Macht begleitete Gesetze, denen

9 ZeF B 71, 08: 370 .

10 ZeF B 111, 08: 386.

$11 \mathrm{Vgl}$. ZeF B 38, 08: 357.

12 MS 06: 350.

13 Ibid.

14 Vgl. ZeF B 38, 08: 357. 
sich jeder Staat unterwerfen müsste, gegründetes Völkerrecht (nach der Analogie eines bürgerlichen oder Staatsrechts einzelner Menschen) möglich. [...] Aber solchen Zwangsgesetzen, wird man sagen, werden sich Staaten doch nie unterwerfen; und der Vorschlag zu einem allgemeinen Völkerstaat, unter dessen Gewalt sich alle einzelne Staaten freiwillig bequemen sollen, um seinen Gesetzen zu gehorchen, mag in der Theorie eines Abt von St. Pierre, oder eines Rousseau, noch so artig klingen, so gilt er doch nicht für die Praxis : wie er denn auch von grossen Staatsmännern, mehr aber noch von Staatsoberhäuptern, als eine pedantisch-kindische aus der Schule hervorgetretene Idee, jederzeit ist verlacht worden.

Ich meinerseits vertraue dagegen doch auf die Theorie, die von dem Rechtsprincip ausgeht, wie das Verhältniss unter Menschen und Staaten seyn soll, und die den Erdengöttern die Maxime anpreist, in ihren Streitigkeiten jederzeit so zu verfahren, dass ein solcher allgemeiner Völkerstaat dadurch eingeleitet werde, und ihn also möglich (in praxi), und dass er seyn kann, anzunehmen; - zugleich aber auch (in subsidium) auf die Natur der Dinge, welche dahin zwingt, wohin man nicht gerne will (fata volentem ducunt, nolentem trahunt). Bei dieser letzteren wird dann auch die menschliche Natur mit in Anschlag gebracht : welche, da in ihr immer noch die Achtung für Recht und Pflicht lebendig ist, ich nicht für so versunken im Bösen halten kann, oder will, dass nicht die moralisch praktische Vernunft nach vielen misslungenen Versuchen endlich über dasselbe siegen, und sie auch als liebenswürdig darstellen sollte. So bleibt es also auch in kosmopolitischer Rücksicht bei der Behauptung: Was aus Vernunftgründen für die Theorie gilt, das gilt auch für die Praxis. ${ }^{15}$

Diese Schlusssätze bringen nun aber keine deutliche Lösung für die Problematik des Verhältnisses zwischen Weltstaat und Völkerbund. Sie lösen zwar einen Teil des Problems, verdunkeln aber einen anderen. Einerseits soll nun klar sein, dass irgendwelche empirisch-praktischen Gründe, die gegen den als Weltstaat aufgefassten Völkerstaat angeführt werden können, nach Kant nie ausreichen können, um die Idee als an sich unproblematische Vorstellung der Vernunft zu verwerfen. Andererseits aber verdunkelt diese Passage, dass die Idee eines Weltstaates tatsächlich verworfen werden soll. Und wenn nicht aus empirisch-praktischen, dann wohl aus nicht-empirischen, d.h. vernünftigen Gründen.

In der Tat ist die Idee eines Weltstaates vernunftwidrig, weil sie aus der völkerrechtlichen Perspektive widersprüchlich ist. Und genau das hatte Kant nun auch schon in seinem Gemeinspruch-Aufsatz, trotz dessen (oben zitierten) Schluss, angedeutet. Einige Absätze zuvor hieß es nämlich:

So wie allseitige Gewalttätigkeit und daraus entspringende Not endlich ein Volk zur Entschließung bringen mußte, sich dem Zwange, den ihm die Vernunft selbst als Mittel vorschreibt, nämlich dem öffentlicher Gesetze zu unterwerfen und in eine staatsbürgerliche Verfassung zu treten: so muß auch die Not aus den beständigen Kriegen, in welchen wiederum Staaten einander zu schmälern oder zu unterjochen suchen, sie zuletzt dahin bringen, selbst wider Willen, entweder in eine weltbürgerliche Verfassung zu treten; oder ist ein solcher Zustand eines allgemeines Friedens [...] auf einer andern Seite der Freiheit noch gefährlicher, indem er den schrecklichsten Despotismus herbeiführt, so muß sie diese Not doch zu einem Zustande zwingen, der zwar kein weltbürgerliches gemeines Wesen unter einem Oberhaupt, aber doch ein rechtlicher Zustand der Föderation nach einem gemeinschaftlich verabredeten Völkerrecht ist. ${ }^{16}$

Hier bleibt es offen, ob der Versuch, in eine weltbürgerliche Verfassung zu treten, zu dem „schrecklichsten Despotismus“ führt. Dass das tatsächlich wahrscheinlich ist, erklärt Kant dann in Zum ewigen Frieden, wo die zwei Alternativen (Weltstaat und Völkerbund) nicht mehr lediglich in einer entweder-oder-Konstruktion nebeneinander gestellt werden, sondern die Idee eines Weltstaates wegen ihrer sowohl begrifflichen als völkerrechtlichen Widersprüchlichkeit bei der Realisierung des Friedens ausdrücklich als unvernünftig ausgewiesen wird. Ein solcher Staat besäße nämlich ein Gewaltmonopol und dies würde bedeuten, dass die verschiedenen Völker eine, Weltstaatsmacht‘ anerkennen. Der Begriff eines solchen Völkerstaates ist schon widersprüchlich, ,weil ein jeder Staat das Verhältnis eines Oberen (Gesetzgebenden) zu einem Unteren (Gehorchenden, nämlich dem Volk) enthält, viele Völker aber in einem Staate nur ein Volk ausmachen würden, 
welches [...] der Voraussetzung widerspricht. “17 Es ginge hier mit anderen Worten nicht mehr um eine völkerrechtliche Lösung, da die Völker im Weltstaat zu einem Volk zusammenschmelzen würden:

Die Idee des Völkerrechts setzt die Absonderung vieler von einander unabhängiger benachbarter Staaten voraus, und, obgleich ein solcher Zustand an sich schon ein Zustand des Krieges ist [...]: so ist doch selbst dieser, nach der Vernunftidee, besser als die Zusammenschmelzung derselben, durch eine die andere überwachsende, und in eine Universalmonarchie übergehende Macht, weil die Gesetze mit dem vergrösserten Umfange der Regierung immer mehr an ihrem Nachdruck einbüßen, und ein seelenloser Despotism, nachdem er die Keime des Guten ausgerottet hat, zuletzt doch in Anarchie verfällt. ${ }^{18}$

Dass Kant dann in der Rechtslehre schreibt: ,so ist der ewige Friede [...] freilich eine unausführbare Idee“"19 , bezieht sich meines Erachtens nur auf diese erste, als völkerrechtliche widersprüchliche Lösung. Die kantische Argumentation im $\S 61$ der Rechtslehre könnte dann wie folgt zusammenfasst werden: Versucht man den ewigen Frieden zu realisieren als notwendiger oder peremptorischer, d.h. in seiner Möglichkeit unbezweifelbarer Frieden, dann müsste man versuchen, einen als Weltstaat aufgefassten Völkerstaat zu errichten; aber da wegen der Natur der Völker und ihrer Rechte nicht einzusehen ist, wie dies anders als mit kriegerischen (oder wenigstens kriegsähnlichen) Maßnahmen gelingen kann, verwandelt sich die Idee eines ewigen Friedens in eine nichtrealisierbare Idee. Der Weltstaat, der für das rein abstrakte Denken die folgerichtige Lösung für das Problem des ewigen Friedens ist, erweist sich im inhaltlich-vernünftigen - d.h. mit der Natur des Menschen, der Völker, der Erde und der Regierungsweisen rechnenden, also nicht mehr rein abstrakten - Denken als nicht imstande, den Frieden zu garantieren.

Gerade wegen des Scheiterns der Idee einer Weltrepublik und spätestens dann, wenn die Umsetzung des Friedensideals nicht als vernunftwidrig gelten soll, wird zweitens die Idee eines freiwilligen Völkerbundes gebildet. In diesem Fall wird von den Staaten keine oberste gesetzgebende Gewalt anerkannt und es wird also auch kein Völkerstaat gegründet. Wenn nun aber jeder Staat sagt: „Es soll kein Krieg zwischen mir und andere Staaten sein, obgleich ich keine oberste gesetzgebende Gewalt erkenne, die mir mein, und der ich ihr Recht sichere" ${ }^{\text {"20 }}$, so ist es nach Kant nicht zu verstehen, worauf dann das Vertrauen, dass die Rechte jedes Staates gesichert werden, gegründet sein sollte, ,wenn es nicht das Surrogat des bürgerlichen Gesellschaftsbundes, nämlich der freie Föderalism ist, den die Vernunft mit dem Begriffe des Völkerrechts notwendig verbinden muß, wenn überall etwas dabei zu denken übrigbleiben soll.“21 Gerade weil der Weg zur Weltrepublik aus völkerrechtlichen Gründen illusorisch ist, wenn es sich, wie die Idee des ewigen Friedens vorschreibt, um einen friedlichen Weg handeln soll, muss ein Surrogatweg gebildet werden, nämlich ein Völkerbund als „Friedensbund“. Nur deshalb also geht es um ein Surrogat, weil diese Idee des Völkerbundes bei einer rein abstrakten Betrachtung nicht als erste Idee aufkommt.

Wie Kant im zweiten Definitivartikel ausführt, beschäftigt sich ein solcher Bund nicht mit der Erwerbung „,irgendeiner Macht des Staats“, sondern geht lediglich ,auf Erhaltung und Sicherung der Freiheit eines Staats für sich selbst und zugleich anderer verbündeten Staaten, ohne daß diese doch sich deshalb [...] öffentlichen Gesetzen und einem Zwange unter denselben unterwerfen dürfen.“22 In der Rechtslehre wird ein solcher Friedensbund dann auch ,,permanente[r] Staatenkongre ${ }^{\text {‘23 }}$ genannt. Wichtig, ist, dass der Beitritt zu diesem Kongress eine freie Entscheidung der 
jeweiligen Staaten sein soll und dass es um eine ,zu aller Zeit ablösliche Zusammentretung verschiedener Staaten" ${ }^{\text {"24 }}$ geht. Nur durch das Anstreben dieser zweiten Lösung als Surrogat für die Weltrepublik kann ,die Idee eines zu errichtenden öffentlichen Rechts der Völker, ihre Streitigkeiten auf zivile Art, gleichsam durch einen Prozeß, nicht auf barbarische (nach Art der Wilden), nämlich durch Krieg zu entscheiden, realisiert werden. “25

Am Ende des zweiten Definitivartikels wird der besprochene Sachverhalt von Kant nochmals zusammenfassend wiederholt:

\begin{abstract}
Für Staaten im Verhältnisse untereinander kann es nach der Vernunft keine andere Art geben, aus dem gesetzlosen Zustande, der lauter Krieg enthält, herauszukommen, als daß sie, ebenso wie einzelne Menschen, ihre wilde (gesetzlose) Freiheit aufgeben, sich zu öffentlichen Zwangsgesetzen bequemen und so einen (freilich immer wachsenden) Völkerstaat (civitas gentium), der zuletzt alle Völker der Erde befassen würde, bilden. Da sie dieses aber nach ihrer Idee vom Völkerrecht durchaus nicht wollen, mithin, was in thesi richtig ist, in hypothesi verwerfen, so kann an die Stelle der positiven Idee einer Weltrepublik (wenn nicht alles verloren werden soll) nur das ne gative Surrogat eines den Krieg abwehrenden, bestehenden und sich immer ausbreitenden B undes den Strom der rechtscheuenden, feindseligen Neigung aufhalten, doch mit beständiger Gefahr ihres Ausbruchs. ${ }^{26}$
\end{abstract}

Man kann nun fragen, ob diese „,beständige Gefahr“ eines Ausbruchs der „rechtscheuenden, feindseligen Neigung“", sogar im Falle der Realisierung eines Friedensbundes, nicht gerade die Unewigkeit des Friedens garantiert. Ist das der Grund dafür, dass Kant sich in der Friedensschrift genötigt sieht, den ewigen Frieden auch extern, d.h. unabhängig vom menschlichen Willen zu garantieren?

\title{
3. DIE GARANTIE DES EWIGEN FRIEDENS
}

In den Definitivartikeln geht es vor allem darum zu zeigen, welche Vorschriften die Menschen befolgen sollten, damit völkerrechtliche Streitfragen unparteilich entschieden werden können und der etablierte Frieden nicht gestört wird. Im ersten Zusatz, „Von der Garantie des ewigen Friedens“, geht es nun darum zu zeigen, wie die Natur selbst den Frieden garantiert, indem sie die Menschen sozusagen zwingt, sich auf gewisse Art zu organisieren. Kant unterscheidet zwei Schritte in seinem Beweis, dass die Natur selbst den Frieden gewährleistet. Erstens beschreibt er den Zustand ,den die Natur für die auf ihrem großen Schauplatz handelnde Personen veranstaltet

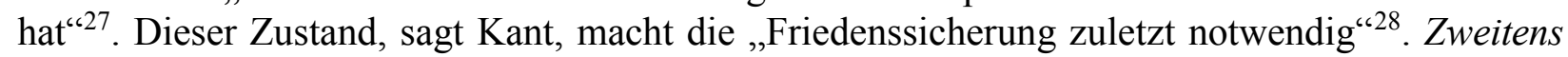
führt er aus, wie die Natur diese Friedenssicherung notwendig macht. An dieser Aufteilung in zwei Schritten werde ich mich auch bei der folgenden Besprechung des ersten Zusatzes halten.

Zunächst muss man bedenken, dass Kant unter Natur durchaus etwas anderes versteht als wir. Bei Natur denken wir heute fast ausschließlich an die Erdoberfläche und die Fauna und Flora, die sich darauf befinden. Bei Kant ist der Begriff noch viel breiter gefasst. Kant unterscheidet zwischen der Natur in sensu formali, die das Wesen einer Sache ausdrückt, und der Natur in sensu materiali, die einen bestimmten Objektbereich betrifft, nämlich die Totalität der Objekte der Sinne. Zum Beispiel ist in der Kritik der reinen Vernunft mit Natur fast immer innere und äußere Natur gemeint. Auch wenn Kant in der Friedensschrift von Natur redet, müssen wir diesem Wort eine weniger beschränkte Bedeutung geben, als es heute hat. Demzufolge ist Natur im materiellen Sinne alles, was Gegenstand der empirischen Erkenntnis ist, also nicht nur Erde, Erdoberfläche (Gebirge, Meere, usw.), Fauna und Flora, sondern auch der Mensch als tierähnliches Wesen und auch das menschliche Verhalten, insofern es empirischen Gesetzen unterworfen ist. Diese in die Richtung der menschlichen Natur gehende Ordnung bei der Aufzählung der Objekte der Natur -

24 Ibid., 351.

25 Ibid.

26 ZeF B 37f., 08: 357.

27 ZeF B 52, 08: 362.

28 Ibid., $362 \mathrm{f}$. 
Erde, Erdoberfläche, Fauna \& Flora, Mensch, menschliches Verhalten - kann man auch in Kants Argumentation selbst unterscheiden.

Qua Erde ist die Natur kugelförmig, das heißt, dass ihre Oberfläche nicht unendlich, also begrenzt, ist. Qua Erdoberfläche ist die Natur so organisiert, dass der Mensch in allen Erdgegenden leben kann. Das hängt damit zusammen, dass die Natur qua Fauna und Flora das Leben der Menschen sogar in den zunächst als menschenfeindlich empfundenen Gegenden ermöglicht, was nach Kant Bewunderung erregen muss:

$\mathrm{Da}$ in den kalten Wüsten am Eismeer noch das Moos wächst, welches das Rentier unter dem Schnee hervorscharrt, um selbst die Nahrung oder auch das Angespann des Ostjaken oder Samojeden zu sein; oder daß die salzigen Sandwüsten doch noch dem Kamel, welches zu Bereisung derselben gleichsam geschaffen zu sein scheint, um sie nicht unbenutzt zu lassen, enthalten, ist schon bewundernswürdig. Noch deutlicher aber leuchtet der Zweck hervor, wenn man gewahr wird, wie außer den bepelzten Tieren am Ufer des Eismeeres noch Robben, Walrosse, Walfische an ihrem Fleische Nahrung und mit ihrem Tran Feuerung für die dortige Anwohner darreichen. Am meisten aber erregt die Vorsorge der Natur durch das Treibholz Bewunderung, was sie [...] diesen gewächslosen Gegenden zubringt, ohne welches Material sie weder ihre Fahrzeuge und Waffen noch ihre Hütten zum Aufenthalt zurichten könnten; wo sie dann mit dem Kriege gegen die Tiere genug zu tun haben, um unter sich friedlich zu leben. ${ }^{29}$

Nun ist die Natur qua menschliches Tier kriegslustig: „Der Krieg selbst bedarf keines besondern Bewegungsgrundes, sondern scheint auf die menschliche Natur gepfropt zu sein, und sogar als etwas Edles, wozu der Mensch durch den Ehrtrieb, ohne eigennützige Triebfedern, beseelt wird“30. Und nur durch Krieg kommt es, dass die Menschen ,allerwärts hin, selbst in die unwirtbarsten Gegenden getrieben [wurden], um sie zu bevölkern“"31. Insofern hat die Natur dafür gesorgt, dass die Menschen nicht nur überall auf Erden leben könnten, sondern auch ,allerwärts leben sollten, wenngleich wider ihre Neigung, und selbst ohne daß dieses Sollen zugleich einen Pflichtbegriff voraussetzte, der sie hierzu vermittelst eines moralischen Gesetzes verbände“ ${ }^{\text {(32 }}$.

Wenn nun die Menschen alle Erdgegenden bevölkern, ist die Frage, wie die Natur ausgehend von diesem Zustand die Friedenssicherung notwendig macht. Bei diesem zweiten Schritt seiner Argumentation geht es Kant darum, zu zeigen wie die Natur qua Mensch (d.h. die empirische menschliche Natur) dafür sorgt, dass dasjenige, was der Mensch in Folge seiner nicht-empirischen Verfassung, d.h. nach dem moralischen Gesetz, tun sollte (um den ewigen Frieden näher zu bringen), sowieso getan wird - auch wenn es nicht aus Pflicht geschieht. Die empirische menschliche Natur (als menschliche Neigung) versichert dies nun ,nach allen drei Verhältnissen des öffentlichen Rechts, des Staats-, Völker- und weltbürgerlichen Rechts “33.

Es ist nicht ausgeschlossen, dass eine Gruppe von Menschen friedlich zusammenleben kann, ohne sich einem öffentlich gesetzlichen äußeren Zwang zu unterwerfen. In einem solchen „,natürlichen Zustand" wäre dann nicht von Ungerechtigkeit die Rede, aber doch von Rechtlosigkeit. Da es auch im Naturzustand immer schon provisorische Erwerbungen gibt, droht dort ständig die Gefahr, dass das (provisorische) Recht auf diese Erwerbungen bestritten wird. In einem Zustand der Rechtlosigkeit könnte man sich in diesem Fall nicht an einen kompetenten Richter wenden. Aber, so Kant, auch wenn ein Volk

nicht durch innere Mißhelligkeit genötigt würde, sich unter den Zwang öffentlicher Gesetze zu begeben, so würde es doch der Krieg von außen tun, indem nach der vorher erwähnten Naturanstalt [die Verbreitung der Menschen

29 ZeF B 53, 08: 363.

30 ZeF B 57f., 08: 365.

$31 \mathrm{ZeF}$ B 52, 08: 363 .

32 ZeF B 56f., 08: 364. Für das Argument ist es nicht erforderlich, dass die Menschen tatsächlich ,allerwärts auf Erden“"leben können. Vielmehr muss die Erde überall dort, wo sie bewohnbar ist, auch bewohnt sein. Der Nordpol dürfte dann als unbewohnbare Erdgegend gelten. Siehe auch Eberl / Niesen 2011, S. 273; Laberge 1995, S. 155; und Piché 2015.

33 ZeF B 59, 08: 365. 
auf die ganze Erde - H.B.] ein jedes Volk ein anderes es drängende Volk zum Nachbar vor sich findet, gegen das es sich innerlich zu einem Staat bilden muß, um als Macht gegen diesen gerüstet zu sein. ${ }^{34}$

Wichtig ist, dass der Staat dabei als Resultat der selbstsüchtigen Neigungen der Menschen errichtet wird. Er kann also als Ergebnis eines Interessenkalküls hervortreten. Das macht Kant deutlich mit dem bekannten Gedankenexperiment eines Volkes von Teufeln. Auch für diese Teufel wäre das Problem der Staatserrichtung auflösbar, wenn sie nur Verstand hätten. Wegen der größeren Probabilität ihres Überlebens inmitten von Volksgenossen, die einander prinzipiell unfriedlich gesinnt sind, würden selbst Teufel bereit sein, sich unter die Zwangsgesetze einer Staatsordnung zu begeben.

Was das Völkerrecht angeht, kommt Kant zurück auf das Ergebnis des zweiten Definitivartikels. Dort hieß es, wie oben ausführlich besprochen wurde, dass die Gründung eines obermächtigen Weltstaates nicht mit der Idee eines Weltfriedens in Einklang gebracht werden kann. Das kann nur ein Friedensbund, der die Staaten frei lässt. Als bloße Idee wäre die Weltrepublik zwar die folgerichtige Lösung, aber wegen ihrer Widersprüchlichkeit im Kontext der Friedensidee als vom Volk getragenes Ideal muss stattdessen ein vernünftiges Surrogat - der Friedensbund - verwirklicht werden. Obgleich das Nebeneinanderbestehen vieler unabhängiger benachbarter Staaten kein Zustand des ewigen Friedens sein kann, schreibt Kant, ist dieser Zustand doch ,nach der Vernunftidee besser als die Zusammenschmelzung derselben durch eine die anderen überwachsende und in eine Universalmonarchie übergehende Macht. “35

Wenn man an eine hierarchische Stufenleiter der politischen Strukturen der Welt denkt, gibt es meines Erachtens für Kant einen Unterschied zwischen einer Universalmonarchie und einer Universalrepublik. Eine solche Behauptung ist allerdings nicht unproblematisch. Im ersten Definitivartikel hat Kant nämlich nicht nur die Republik als ideale Regierungsart bestimmt, er hat dieses Ideal auch mit der Monarchie als ideale Staatsform verbunden. Das gilt aber nur für den Fall, dass die ausführende Gewalt von der gesetzgebenden Gewalt unterschieden ist. Zwar ist die republikanische Monarchie für Kant das Ideal, aber es hat diese Staatsform noch nicht gegeben, weil noch keine Monarchie republikanisch gewesen ist. Wenn Kant nun hier im ersten Zusatz über eine Universalmonarchie redet, denkt er also nicht an einer Monarchie mit Gewaltenteilung. Und anders als eine Weltrepublik wäre eine Universalmonarchie ohne republikanische Regierungsart notwendig (und nicht nur wahrscheinlich) despotisch und gewalttätig. Trotz dessen gibt Kant ein Argument gegen die Realisierbarkeit einer Universalmonarchie, das sich zugleich auch auf die Unrealisierbarkeit des Vernunftideals - die Weltrepublik - beziehen lässt. Alleinherrscher mögen das Verlangen haben, viele Staaten zusammenzuschmelzen bis es eine Universalmonarchie gibt, und vielleicht denken sie dabei sogar an die Etablierung des ewigen Friedens. Aber die Natur der Menschen verhindert dies: Völker werden von der Vermischung abgehalten durch „die Verschiedenheit der Sprachen und der Religionen“36.

In Bezug auf das Weltbürgerrecht garantiert die natürliche Neigung der Menschen, dass sie sich vor allem um die Eigennützlichkeit ihres Handelns kümmern, dass sie bevorzugen, das benachbarte Volk als Handelspartner statt als Kriegsfeind zu betrachten. Da die Geldmacht die zuverlässigste der der Staatsmacht untergeordneten Mächte ist, werden die Menschen naturgemäß dazu neigen, den Frieden zu befördern und den Krieg durch Vermittlungen abzuwehren, ,gleich als ob sie deshalb im beständigen Bündnisse ständen““37. Der Handelsgeist, „der mit dem Kriege nicht zusammen bestehen kann" ${ }^{638}$, befördert den permanenten Friedenskongress, auch ohne dass 
sich jemand jemals durch Triebfedern der Moralität dazu gedrungen sieht. Abschließend erklärt Kant dann noch einmal die Art der in der Natur selbst verkörperte Garantie des ewigen Friedens:

Auf die Art garantiert die Natur durch den Mechanism in den menschlichen Neigungen selbst den ewigen Frieden; freilich mit einer Sicherheit, die nicht hinreichend ist, die Zukunft desselben (theoretisch) zu weis sagen, aber doch in praktischer Absicht zulangt und es zur Pflicht macht, zu diesem (nicht bloß chimärischen) Zwecke hinzuarbeiten. ${ }^{39}$

Die von der Natur gegebene Garantie ist nun gewiss nicht absolut, aber sie reicht, um den Begriff des ewigen Friedens nicht als leere und unausführbare Idee betrachten $\mathrm{zu}$ müssen. Und mehr braucht man nicht, um sich seine Beförderung zur Pflicht machen zu können.

\section{DER EWIGE FRIEDE ALS INTERESSIERTE ANNAHME}

In der Rechtslehre gibt es keinen Abschnitt mehr über die ,Garantie des ewigen Friedens'. Kant führt 1796 eigentlich nur ein Argument an, das sich auch im ersten Zusatz der Friedensschrift finden lässt. Im $\S 43$ schreibt er, dass ,,der Erdboden eine nicht gränzenlose, sondern sich selbst schließende Fläche ist ${ }^{640}$, was im $\S 62$ dann nochmal als von der Natur garantierter Zustand vorgestellt wird: „Die Natur hat sie [die Völker - H.B.] alle zusammen (vermöge der Kugelgestalt ihres Aufenthalts, als globus terraqueus) in bestimmte Grenzen eingeschlossen“"41. Trotzdem glaube ich nicht, dass Kant dort die Frage der Realisierbarkeit des ewigen Friedens wirklich anders beantwortet als in der Friedensschrift. In der Rechtslehre geht es vor allem um die Realisierung des ewigen Friedens als Pflicht des Menschen und der Völker, unabhängig davon, ob es auch empirische Zeichen gibt, die darauf hinweisen, dass ein solcher Frieden möglich ist. Es kann weder bewiesen werden, dass der ewige Frieden möglich ist, noch dass er unmöglich ist.

Die Garantie in der Friedensschrift dagegen scheint darauf ausgelegt zu sein, zu beweisen, dass der ewige Frieden möglich ist, auch wenn Kant dadurch nicht glaubt, bewiesen zu haben, dass er jemals auch wirklich realisiert wird. Aber um welche Art der Möglichkeit würde es sich da handeln? Streng genommen müsste es sich um eine metaphysische Möglichkeit handeln, weil wir vom ewigen Frieden keine Anschauung haben. Nach Kants Theorie der Modalitäten können wir über die Möglichkeit des ewigen Friedens nichts behaupten - auf keinen Fall ist die reale Möglichkeit bewiesen (obwohl Kant die objektive Realität der Idee für bewiesen hält). Aber auch wenn wir weder Möglichkeit noch Unmöglichkeit des ewigen Friedens beweisen können, bleibt es Pflicht, zu versuchen, ihn zu realisieren.

Wenn jemand nicht beweisen kann, daß ein Ding ist, so mag er versuchen zu beweisen, daß es nicht ist. Will es ihm mit keinem von beiden gelingen (ein Fall, der oft eintritt), so kann er noch fragen: ob es ihn interessiere, das eine oder das andere (durch eine Hypothese) anzunehmen, und dies zwar entweder in theoretischer, oder in praktischer Rücksicht, d.i. entweder um sich bloß ein gewisses Phänomen [...] zu erklären, oder um einen gewissen Zweck zu erreichen, der nun wiederum entweder pragmatisch [...] oder moralisch, d.i. ein solcher Zweck sein kann, den sich zu setzen die Maxime selbst Pflicht ist. - Es versteht sich von selbst: daß nicht das Annehmen (suppositio) der Ausführbarkeit jenes Zwecks, welches ein bloß theoretisches und dazu noch problematisches Urteil ist, hier zur Pflicht gemacht werde, denn dazu (etwas zu glauben) gibt's keine Verbindlichkeit, sondern das Handeln nach der Idee jenes

39 ZeF B 66, 08: 368.

40 MS 06: 311.

41 MS 06: 352. 
Zwecks, wenn auch nicht die mindeste theoretische Wahrscheinlichkeit da ist, daß er ausgeführt werden könne, dennoch aber seine Unmöglichkeit gleichfalls nicht demonstriert werden kann, das ist es, wozu uns eine Pflicht obliegt. ${ }^{42}$

Im Rahmen unserer theoretischen Erkenntnis wissen wir nicht, ob der ewige Frieden ein Ding oder ein Unding ist, weil metaphysische Möglichkeit von uns nicht bewertet werden kann. In praktischer Hinsicht aber ist es Pflicht, den Frieden anzustreben und das Können geht hier direkt aus dem Sollen hervor: Wir können auf den ewigen Frieden hinarbeiten, weil wir es sollen. Für den Menschen als moralisches Wesen leuchtet diese Pflicht von selbst ein, da wir aus reiner Vernunft wissen, dass der ewige Frieden der Endzweck des ganzen Völkerrechts ist.

\section{LITERATUR}

Buhr, Manfred / Dietzsch, Steffen (Hrsg.), 1984: Immanuel Kant. Zum ewigen Frieden. Mit Texten zur Rezeption 1796-1800. Leipzig.

Eberl, Oliver / Niesen, Peter, 2011: Immanuel Kant. Zum ewigen Frieden. Kommentar. Berlin.

Fichte, Johann Gottlieb, 1796: Rezension. In: Niethammer, Friedrich Immanuel / Fichte, Johann Gottlieb (Hrsg.): Philosophisches Journal, 4, S. 81-92.

Kodalle, Klaus-Michael (Hrsg.), 1996: Der Vernunftfrieden. Kants Entwurf im Widerstreit. Würzburg.

Laberge, Pierre, 1995: Von der Garantie des ewigen Friedens. In: Höffe, Otfried (Hrsg.): Immanuel Kant. Zum ewigen Frieden. Berlin, S. 149-170.

Laschet, Oliver, 2011: Metaphysik und Erfahrung in Kants praktischer Philosophie. Freiburg / München.

Piché, Claude, 2015 : La Rotondité de la Terre: une chance pour la paix. In: Kant-Studien, 106, S. 371-397. 